\title{
Toxicity Test
}

National Cancer Institute

\section{Source}

National Cancer Institute. Toxicity Test. NCI Thesaurus. Code C17755.

Controlled laboratory tests to determine the toxicity of a chemical to an organism in terms of specific chemical concentrations. 